\title{
Studies on coexistence of $m e c$ gene, IS256 and novel sasX gene among human clinical coagulase-negative staphylococci
}

\author{
K. R. Soumya ${ }^{1} \cdot$ Sheela Sugathan $^{2} \cdot$ Jyothis Mathew $^{1} \cdot$ E. K. Radhakrishnan ${ }^{1}$ (I)
}

Received: 27 August 2016/ Accepted: 14 October 2016/Published online: 31 October 2016

(c) The Author(s) 2016. This article is published with open access at Springerlink.com

\begin{abstract}
Methicillin-resistant coagulase-negative staphylococci (MRCoNS) are major nosocomial pathogens which cause device-related infections. Presence of various virulence factors along with methicillin resistance favor improved CoNS pathogenicity and their dissemination among population. In the present study, mec gene prevalence was analyzed along with SCC mec diversity among 55 human clinical CoNS isolates. PCR screening of insertion sequence (IS256) was also conducted to elucidate their association with methicillin resistance among selected CoNS species. In addition to this, PCR screening and sequence analysis of novel colonization-associated gene sas X was also carried out. High incidences of mec gene, IS256 and their association have been noted among all of the CoNS species tested. Interestingly, eight CoNS isolates were found to harbor sas X gene including S. epidermidis, $S$. hemolyticus and S. saprophtyicus species. Remarkably they were also found to have the coexistence of mec gene and IS256 in their genome. Increased SCC mec diversity with non-typeable elements was also observed among CoNS isolates. Presence of sas X gene in CoNS with mec gene and insertion sequence 256 and also the identification of non-typeable SCC mec element make the study novel and interesting.
\end{abstract}

Electronic supplementary material The online version of this article (doi:10.1007/s13205-016-0549-9) contains supplementary material, which is available to authorized users.

E. K. Radhakrishnan

radhakrishnanek@mgu.ac.in

1 School of Biosciences, Mahatma Gandhi University, PD Hills (PO), Kottayam 686 560, Kerala, India

2 MOSC Medical College, Kolencherry 682 311, Kerala, India
Keywords Coagulase-negative staphylococci $\cdot$ Methicillin resistance $\cdot$ sas X gene $\cdot$ mec gene $\cdot$ IS256

\section{Introduction}

Coagulase-negative staphylococci (CoNS) are major component of the normal flora of human cutaneous ecosystem. In recent years, CoNS have emerged as frequent causative agents of nosocomial illness (Lo et al. 2010). More than 14 species of CoNS are currently recognized as human pathogens and the frequently encountered species are $S$. epidermidis, S. hemolyticus, S. hominis, and S. saprophyticus (Kloos et al. 1994). The range of infections caused by CoNS include bacteremia, native and prosthetic valve endocarditis, osteomyelitis, pyoarthritis, peritonitis and implanted medical device-related infections (Huebner and Goldmann 1999). The remarkable biofilm formation and ability to acquire and disseminate multidrug resistance have been considered to contribute significantly to the emergence and propagation of CoNS in nosocomial and community settings (Longauerova 2006; Duran et al. 2012). The biofilm formation in CoNS can either be of icaADBC-mediated polysaccharide type or of proteinaceous type (von Eiff et al. 2002) and this reduces bacterial susceptibility to drugs.

As CoNS are part of commensal flora, the presence of drug resistance in them is highly dangerous. The mechanism of resistance to penicillin is mainly dependent on the expression of the mecA gene, which encodes PBP2a transpeptidase with low affinity towards most of the semisynthetic penicillins (Duran et al. 2012). Remarkably $m e c \mathrm{~A}$ gene is present as a part of staphylococcal cassette chromosome mec element (SCC mec). SCC mec composed of $m e c$ gene complex, $c c r$ gene complex and three joining 
(J) regions. Based on the classes of the mec gene complex and the $c c r$ gene type there are 11 different SCC mec types for $S$. aureus which itself highlights its importance. But SCC mec elements are likely to be more diverse in MRCoNS due to continuous generation of new composite cassettes that do not fit within the classification proposed for MRSA (Lo et al. 2010; Lebeaux et al. 2011; Zong et al. 2011). Another genetic element, the insertion sequence IS256 which is mainly involved in phase variation of biofilm forming CoNS has been identified to have coexistence with mec gene (Kozitskaya et al. 2005). Autonomous movement and multiple independent insertions of this element into the staphylococcal chromosome together with point mutations, homologous recombination, and horizontal gene transfer act as driving forces for the generation of novel genetic and phenotypic variants. Role of IS256 in association with methicillin heteroresistance in $S$. aureus and the modulation of methicillin resistance in $S$. sciuri has also been suggested (Maki and Murakami 1997; Couto et al. 2003). Hence studies on coexistence of mec gene and IS256 among CoNS from clinical samples are highly interesting and significant.

Genetic analysis on association of mecA and IS256 with other newly identified virulence factors may provide deeper insight into emerging molecular programming which is being conducted in CoNS. The novel sasX gene with a signal peptide and LPXTG surface anchoring motif identified from $S$. aureus with $\Phi S P \beta$-like prophage is very significant in this context. No other orthologues for this gene have been reported except in S. epidermidis RP62A $\Phi S P \beta$-like prophage which has $95.1 \%$ amino acid identity to the sesI gene associated with the clinical strains with invasive properties. SasX provide advantages to the organism due to its role in increased nasal colonization onto host, immune evasion, virulence, bacterial aggregation, biofilm formation and even attachment to the abiotic surfaces (Holden et al. 2010; Li et al. 2012; Otto 2012). In our previous study, we could identify the presence of $\operatorname{sas} \mathrm{X}$ gene in S. epidermidis (Soumya et al. 2016). Hence in this study, prevalence of sas $\mathrm{X}$ gene was analyzed among methicillin-resistant clinical isolates of coagulase-negative staphylococci to investigate its relation to $m e c \mathrm{~A}$ gene and IS256.

PCR based molecular analysis for the coexistence of mec gene, IS256 and sasX gene and also SCC mec typing was carried out for CoNS from human clinical samples. The result showed remarkable coexistence and relation between selected genes and also presence of untypeable SCC mec elements in CoNS which provide precautions about the likely role of CoNS to further disseminate these to other microorganisms of human microbiome.

\section{Materials and methods}

\section{Strains used in this study}

A total of 100 coagulase-negative staphylococci from different clinical samples including exudates, urine, blood, catheter tips, and sputum were collected from a tertiary care hospital in Ernakulam, Kerala, India. The clinical isolates from nutrient agar slopes were quadrantly streaked on to tryptic soy agar plates and incubated for 24-48 h. The colonies observed as coagulase-negative staphylococci on the basis of colony morphology were selected and these isolates were identified using Gram staining, coagulase test and biochemical tests (unpublished data). Methicillin resistance were initially screened using oxacillin and cefoxitin disc diffusion assays, from these 55 MRCoNS strains were obtained which were further selected for this study. Genomic DNA was extracted using the Bacterial genomic DNA isolation Mini Spin kit (Chromous Biotech, Bangalore) following the manufacturer's instructions. Molecular identification of CoNS species was conducted using Multiplex PCR (MPCR). Here, identification of 55 CoNS were performed by amplifying the $n u c$ and adjacent genes using species specific primers (Hirotaki et al. 2011). 4 CoNS isolates which were not amplified in MPCR were further subjected to 16SrDNA sequencing (Chun and Goodfellow 1995).

\section{PCR screening for mecA gene, IS256 and sasX gene}

Separate PCRs were conducted for the detection of mecA gene, IS256 and sasX. For the detection of mecA, gene the primers used were mecA-F, (5'-GAAATGACTGAACG TCCGAT-3') and mecA-R, (5'-GCGATCAATGTTACCG TAGT-3') to amplify a 154-bp gene fragment (Rohde et al. 2004). The presence of IS256 was screened by using IS256-F (5'-TGAAAAGCGAAGAGATTCAAAGC- $\left.3^{\prime}\right)$ and IS256-R (5'ATGTAGGTCCATAAGAACGGC-3') primers (Ziebuhr et al. 1997).

Amplification of a 522-bp fragment of sas X gene was carried out using the primers sasX-F (5'-AGAATTAGA AGTACGTCTAAATGC $\left.3^{\prime}\right)$ and sasX-R (5'GCTGATTAT GTAAATGACTCAAATG- ${ }^{\prime}$ ) ( $\mathrm{Li}$ et al. 2012). In all the PCR reactions, the $50 \mu \mathrm{L}$ PCR mix contained, $5 \mu \mathrm{L}$ of $10 \times$ PCR buffer, $4 \mu \mathrm{L}$ of dNTPs $(200 \mu \mathrm{M}), 0.5$ units of Taq DNA polymerase, $4 \mu \mathrm{L}$ (5 pmol) of specific primers and $6 \mu \mathrm{L}$ of DNA template. The volume was made up to $50 \mu \mathrm{L}$ with sterile MilliQ water. PCR was conducted on Mycy$\operatorname{cler}^{\mathrm{TM}}$ (Bio-Rad, USA) with initial denaturation for $5 \mathrm{~min}$ at $94{ }^{\circ} \mathrm{C}$, followed by 35 cycles of denaturation at $94{ }^{\circ} \mathrm{C}$ for $30 \mathrm{~s}$, annealing at primer specific temperature for $30 \mathrm{~s}$ 
and elongation at $72{ }^{\circ} \mathrm{C}$ for $1 \mathrm{~min}$, with a final extension at $72{ }^{\circ} \mathrm{C}$ for $4 \mathrm{~min}$. The amplified products were analyzed on $1.5 \%$ of agarose gel and were visualized on UV transilluminator. To confirm the identity of the PCR amplicons and to analyze sequence variations among staphylococcal species, all of the sas X PCR products were further gel purified and sequenced using Big Dye Terminator Sequence Reaction Ready Mix (Applied Biosystem). The sequence data obtained were further subjected to BLAST analysis.

\section{Determination of SCC mec types}

For this, a total of $20 \mathrm{CoNS}$ (four $S$. hominis, three $S$. hemolyticus, one S. sciuri, one S. cohnii, two S. saprophyticus and nine $S$. epidermidis) harboring mec gene were subjected to SCC mec typing using Multiplex SCC mec typing MRSA Detection kit (HiMedia, Mumbai). As per manufacture's protocol the PCR was conducted in Sure cycler 8800 (Agilent) and the obtained amplicons were visualized on $2 \%$ agarose gel.

\section{Results}

From a total of 100 CoNS isolates processed, 55 isolates which showed methicillin resistance on antibiotic sensitivity assay were selected for the study. Among 55 MRCoNS, 51 isolates showed amplification specific for different CoNS species. 31 isolates showed amplification corresponding to $S$. epidermidis (251 bp) followed by 13 S. hemolyticus isolates (434 bp). Amplification of nис gene resulted in 843-bp product for five isolates specific to $S$. saprophyticus, whereas other four isolates showed 177-bp product specific for the species S. hominis. Rest of the MPCR unidentified clinical isolates were subjected to $16 \mathrm{~S}$ rDNA partial sequencing and the results were BLAST analyzed. Two of the isolates were identified with $100 \%$ identity to S. hemolyticus and other two isolates showed maximum identity with $S$. sciuri and with $S$. cohnii. Thus $S$. epidermidis $(n=31)$ showed $56 \%$ distribution followed by $S$. hemolyticus ( $n=13$, $24 \%)$, S. saprophyticus $(n=5,9 \%), S$. hominis $(n=4$, $7 \%), \quad$ S. sciuri $(n=1,2 \%)$, S. cohnii $(n=1,2 \%)$ (Supplementary Figure 1).

\section{PCR screening for mecA gene, IS256 and sas X gene among CoNS clinical isolates}

PCR results confirmed 53 CoNS isolates (96\%) to have mec gene and IS256 individually, whereas 52 (95\%) isolates were found to have the presence of both the genes. Among tested S. epidermidis, 97\% amplified mec gene whereas $94 \%$ were found to be IS256 positive. All of the 13 S. hemolyticus strains harbored IS256 but mec gene absence was observed for one of the $S$. hemolyticus isolate. Rest of the CoNS isolates tested in this study ( $S$. saprophyticus, S. hominis, S. sciuri and S. cohnii) were found to have the coexistence of both the mec gene and IS256 in their genome. Distribution of these genes among CoNS is described in Table 1, Figs. 1 and 2.

Among 55 samples analyzed, eight isolates showed presence of product of $522 \mathrm{bp}$ size corresponding to the size of sas $\mathrm{X}$ gene and hence these were further sequenced, analyzed by BLAST and compared with the sasX gene of S. aureus TW20. Interestingly, these eight isolates also harbored mec gene and IS256 within their genome. Specifically, four $S$. epidermidis isolates, three $S$. hemolyticus and one $S$. saprophyticus were found to possess this gene. Translated sas X gene sequence of three $S$. hemolyticus, two S. epidermidis and one S. saprophyticus showed $100 \%$ identity to the LPXTG sequence of $S$. aureus TW20, whereas other two S. epidermidis isolates showed only 98 and $95 \%$ identities to the reference sequence (Fig. 3).

\section{SCC mec typing using MPCR assay Kit}

SCC mec typing has been carried out for selected isolates from each species using the multiplex kit. Of 20 isolates tested, 16 isolates showed amplification for the mec gene. Only six isolates showed the presence of SCC mec specific amplification pattern. Three $S$. epidermidis belonged to different SCC mec types. Two of the $S$. epidermidis isolates had multiple amplifications at 280bp and $325 \mathrm{bp}$ which indicated the occurrence of both the SCC mec type III and $\mathrm{V}$, respectively, within these isolates, whereas another isolate had an amplification corresponding to SCC mec type III only. One of the $S$. hemolyticus isolates showed the amplification corresponding to the SCC mec type V. In the case of $S$. hominis, one isolate showed amplification corresponding to SCC mec type III and other isolate to type V. (Fig. 4).

\section{Discussion}

Even though coagulase-negative staphylococci were considered as harmless skin commensals, their frequent isolation from various clinical samples has changed its status to nosocomial pathogens. As MRCoNS are widespread in all parts of the world, it represent a serious burden of infection in both nosocomial and community settings. Various factors involved in CoNS pathogenesis are now being explored; major among them is methicillin resistance 
Table 1 Distribution of mec gene, IS256 and sas X gene among CoNS species

\begin{tabular}{lccl}
\hline CoNS species & mec gene & IS256 & sas X gene \\
\hline S. epidermidis & 30 & 29 & 4 \\
S. hemolyticus & 12 & 13 & 3 \\
S. saprophyticus & 5 & 5 & 1 \\
S. hominis & 4 & 4 & - \\
S. sciuri & 1 & 1 & - \\
S. cohnii & 1 & 1 & - \\
\hline
\end{tabular}

and associated virulence determinants (Ibrahem et al. 2009).

In this study, 55 MRCoNS were selected from clinical samples which belonged to six different CoNS species. Among these $30 \mathrm{~S}$. epidermidis were found to carry mec gene. Various reports on methicillin carriage in S. epidermidis showed it to have a distribution rate of $69-84 \%$ (Lebeaux et al. 2011) and is in accordance with the results of current study. In addition, all of the $S$. hominis, $S$. saprophyticus, S. cohnii and S. sciuri isolates were also found to have the presence of mec gene. Human clinical isolates of these species were already demonstrated to have mec gene with diverge SCC mec types (Mombach Pinheiro Machado et al. 2007). Out of the 13 methicillin-resistant $S$. hemolyticus identified in the present study, 12 were identified to have the association of mec gene which confirms their major role in promoting antibiotic resistance phenotypes (Diekema et al. 1999).

Among 20 isolates tested using SCC mec multiplex identification kit, 16 showed mec gene amplification and 6 of the isolates showed the presence of SCC mec elements. Apart from the common classical mecA gene, different variants $m e c \mathrm{~A} 1, m e c \mathrm{~A} 2, m e c \mathrm{C}$ and $m e c \mathrm{C} 2$ have also been reported from CoNS species of both human and animal origin and the polymorphism can expected to have determining effect on its positive result in the PCR screening (Becker et al. 2014). SCC mec diversity in staphylococci is very common and till now almost 11 SCC types and various subtypes has been reported. In this study two S. epidermidis isolates showed amplification for multiple SCC types-III and V, also two S. hominis isolates individually showed the presence of SCC mec type III and V. Another single isolate of $S$. epidermidis showed the presence of SCC mec type III and one S. hemolyticus isolate was found to be PCR positive for SCC mec type V. These results were in accordance with the previous reports suggesting the high prevalence of SCC mec type III, IV and V among CoNS. Non-typeable mec elements are frequent in CoNS and this may be the reason for the absence of SCC mec types among the other CoNS isolates tested in this study (Zong and Lü 2010).

High degree of coexistence of IS256 with mec gene was also identified for all the CoNS species used in this study.
Fig. 1 PCR amplification of mec A gene. Lanes 1-55 - CoNS isolates, $\mathrm{M}$ - DNA molecular weight marker, $\mathrm{C}$ - negative control. mecA gene amplification corresponds to $154 \mathrm{bp}$ product

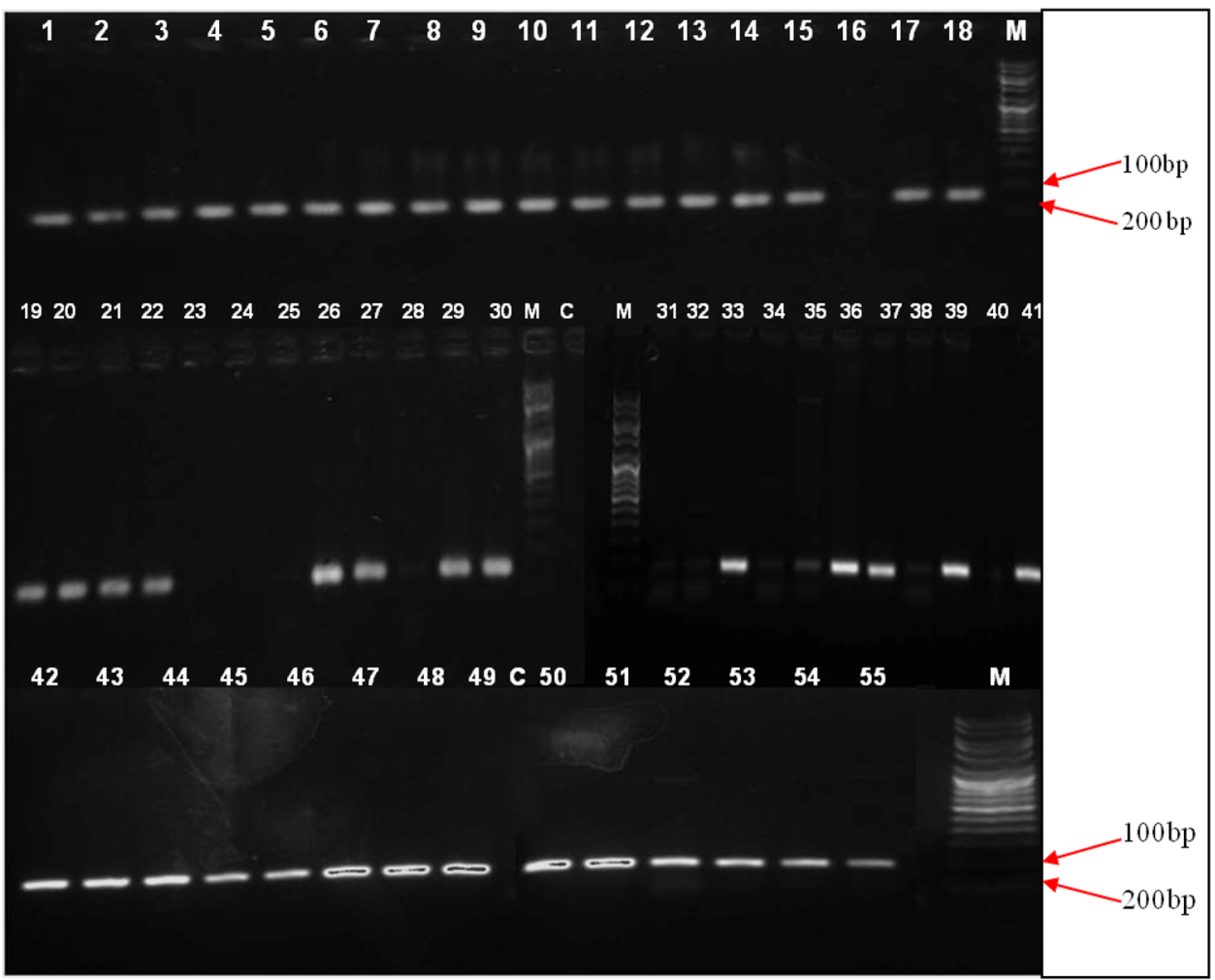


Fig. 2 PCR identification of IS256 among 55 CoNS species. Lanes 1-55 - CoNS isolates, $\mathrm{M}$ DNA molecular weight marker, C - negative control. IS256 amplification is indicated by 1102 bp product
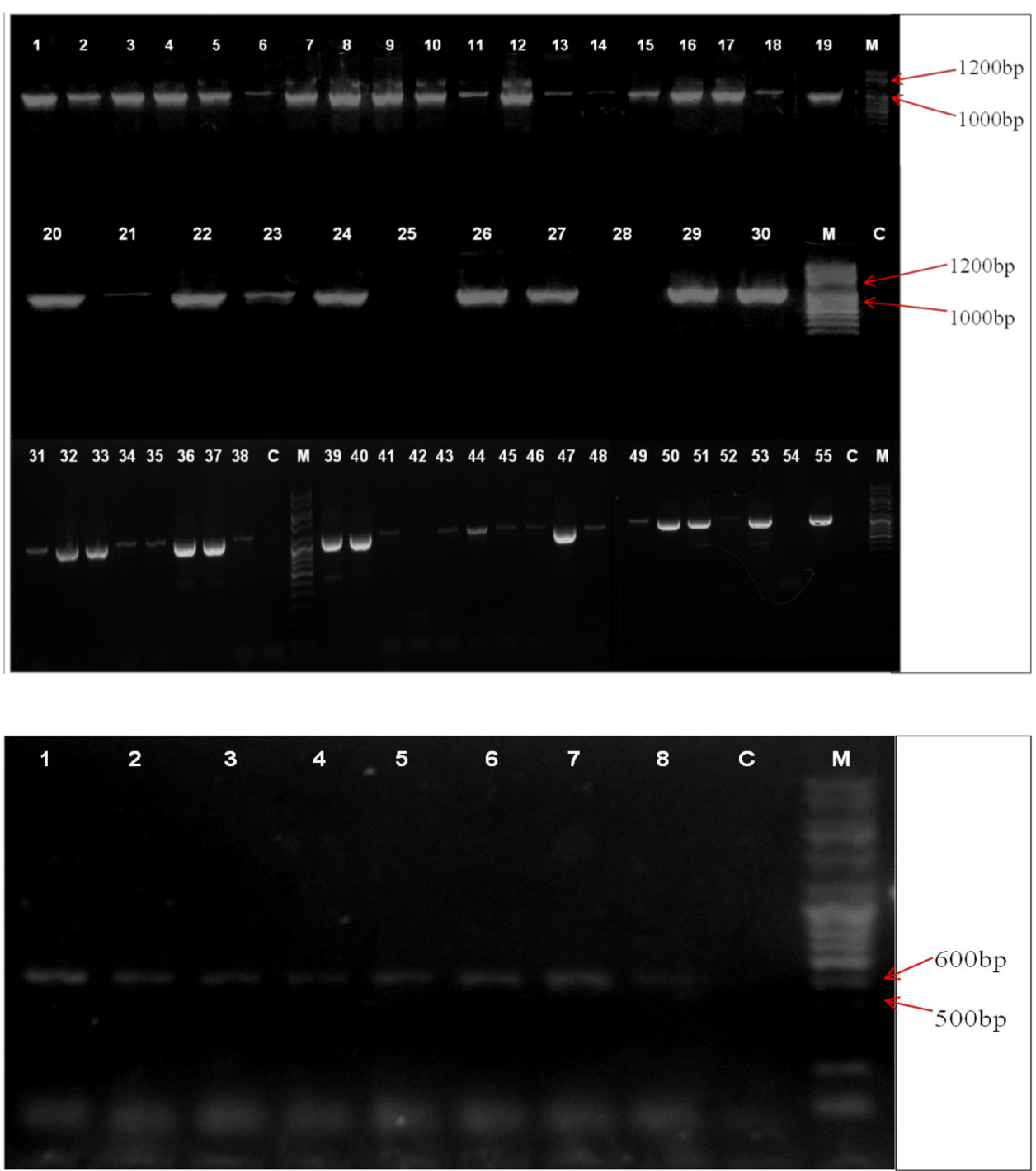

Fig. 3 PCR identification of sas $\mathrm{X}$ gene. Lanes 1-8 represent sas $\mathrm{X}$ gene amplification at 522 bp for CoNS isolates, M DNA molecular weight marker, C - negative control
More specifically, $29 \mathrm{~S}$. epidermidis isolates, $12 \mathrm{~S}$. hemolyticus isolates and all other CoNS isolates were found to carry both IS256 and mec genes in their genome. Overall $95 \%$ of the CoNS isolates showed the association of these genes and the result was in accordance with previous report. IS256 has also been suggested as a good marker gene to differentiate between invasive and noninvasive nature of CoNS isolates due to its frequent association with multidrug-resistant isolates (Mertens and Ghebremedhin 2013). Mechanistically, IS256 transpositions have the potential to create new hybrid promoter with enhanced transcription with concomitant elevation of the methicillin resistance (Maki and Murakami 1997).

Further analysis of coexistence of sas X gene along with IS256 and mec gene showed the presence of novel sasX gene among $S$. epidermidis, $S$. hemolyticus and $S$. saprophyticus species. Its existence in S. epidermidis was already reported in our previous work (Soumya et al.
2016). However, the presence of this gene in $S$. hemolyticus and $S$. saprophyticus species is a novel report and this was confirmed by sequence analysis which showed $100 \%$ percentage identity to the LPXTG motif of $S$. aureus TW20. Even though two of the S. epidermidis isolates were $100 \%$ similar to the reference gene, other two isolates showed only 98 and $95 \%$ similarity. Interestingly the eight sasX-positive isolates identified in this study were also positive for mec gene and IS256 which indicate the possibility to evolve CoNS as potential virulent pathogens. The presence of sas X gene can enhance the colonization property of CoNS and once this occurs $m e c$ gene and IS256 further promote rapid propagation of the organism resulting in serious infections. Also the presence of sas $\mathrm{X}$ gene among different CoNS species identified in the study indicates the possible horizontal transfer of the property which was earlier suspected to occur from S. aureus (Otto 2012). 
Fig. 4 PCR identification of $S C C$ mec types. M - DNA molecular weight marker, $\mathrm{C}$ negative control. Lanes 1-16 CoNS isolates with an amplification at $147 \mathrm{bp}$ corresponding to mec gene. Lanes 4 and 12 SCC mec type III with amplification at $280 \mathrm{bp}$. Lanes 2 and 10 multiple amplification at $280 \mathrm{bp} 325 \mathrm{bp}$ correspond to SCC mec type III and $\mathrm{V}$, respectively. Lanes 11 and 13 amplification at $325 \mathrm{bp}$ indicating the presence of SCC mec type $\mathrm{V}$

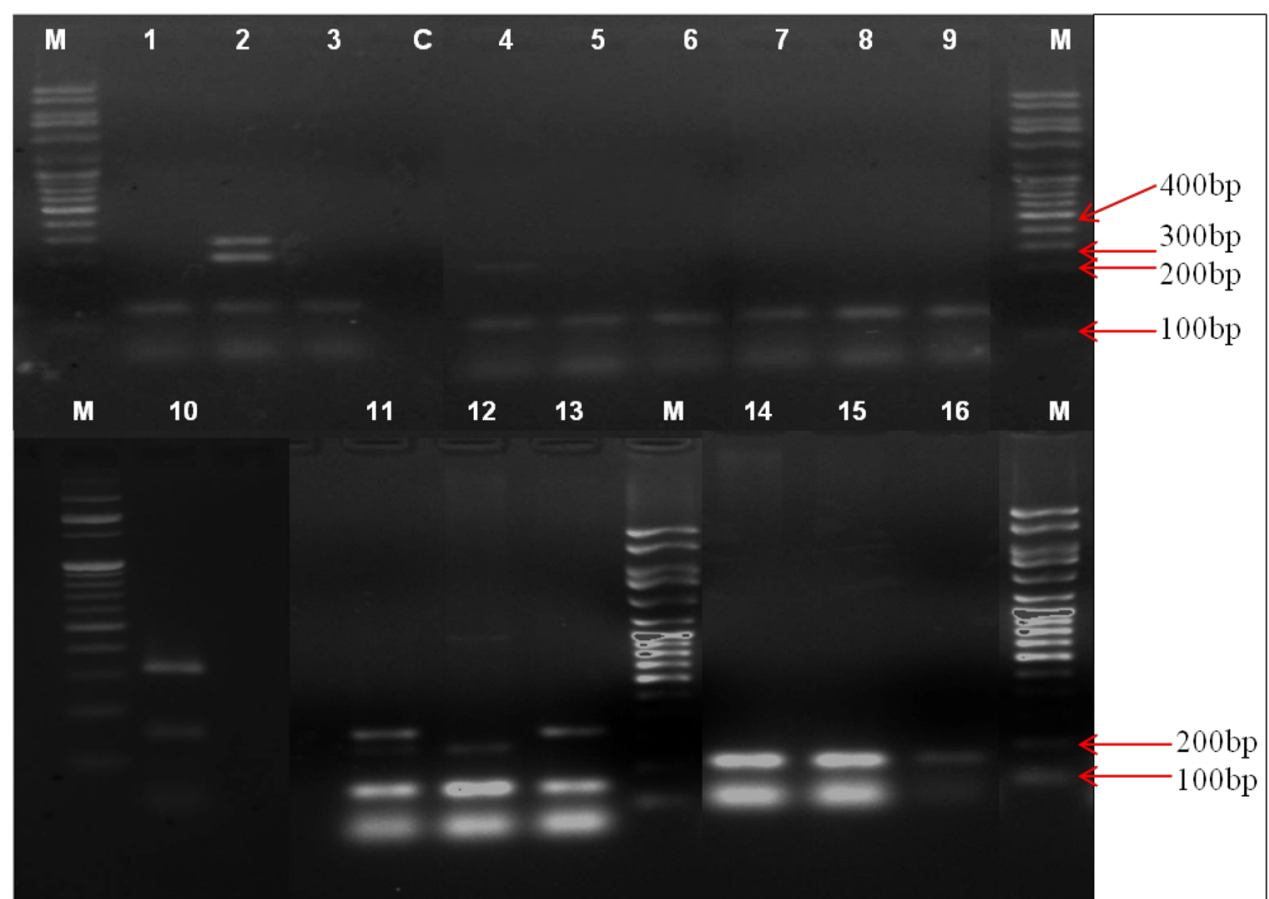

The high degree of coexistence of methicillin resistance and IS256 sequence among different species of CoNS indicates their role in infectious disease. Also the colonization promoting sas $\mathrm{X}$ gene can favor these characteristics which can lead even to their asymptomatic carriage among community settings. These findings highlight the importance of the present work which provide molecular insight into the prevalence of genes which facilitate survival and pathogenesis of CoNS species in the human host which can ultimately contribute to the virulence potential of these dangerous pathogens.

Acknowledgements We gratefully acknowledge Indian Council of Medical Research, Government of India, for the funded project in Coagulase negative staphylococci. Also we thank DBT-MSUB program, School of Biosciences, Mahatma Gandhi University, Kottayam, Kerala, India, for providing instrumentation facility. We thank Dean and laboratory staffs of MOSC Medical College, Kolencherry, Kerala, India.

\section{Compliance with ethical standards}

Conflict of interest The authors declare no conflict of interest.

Open Access This article is distributed under the terms of the Creative Commons Attribution 4.0 International License (http:// creativecommons.org/licenses/by/4.0/), which permits unrestricted use, distribution, and reproduction in any medium, provided you give appropriate credit to the original author(s) and the source, provide a link to the Creative Commons license, and indicate if changes were made.

\section{References}

Becker K, Heilmann C, Peters G (2014) Coagulase-negative staphylococci. Clin Microbiol Rev 27:870-926. doi:10.1128/CMR. 00109-13

Chun J, Goodfellow M (1995) A phylogenetic analysis of the genus Nocardia with 16S rRNA gene sequences. Int J Syst Bacteriol 45:240-245. doi:10.1099/00207713-45-2-240

Couto I, Wu SW, Tomasz A, De Lencastre H (2003) Development of methicillin resistance in clinical isolates of Staphylococcus sciuri by transcriptional activation of the mecA homologue native to the species development of methicillin resistance in clinical isolates of Staphylococcus sciuri by transcript. J Bacteriol 185:645-653. doi:10.1128/JB.185.2.645

Diekema DJ, Pfaller MA, Jones RN et al (1999) Survey of bloodstream infections due to gram-negative bacilli: frequency of occurrence and antimicrobial susceptibility of isolates collected in the United States, Canada, and Latin America for the SENTRY Antimicrobial Surveillance Program, 1997. Clin Infect Dis 29:595-607

Duran N, Ozer B, Duran GG et al (2012) Antibiotic resistance genes \& susceptibility patterns in staphylococci. Indian J Med Res 135:389-396

Hirotaki S, Sasaki T, Kuwahara-arai K, Hiramatsu K (2011) Rapid and accurate identification of human-associated staphylococci by use of multiplex PCR. J Clin Microbiol 49:3627-3631. doi:10. 1128/JCM.00488-11

Holden MTG, Lindsay JA, Corton C et al (2010) Genome sequence of a recently emerged, highly transmissible, multi-antibiotic- and antiseptic-resistant variant of methicillin-resistant Staphylococcus aureus, sequence type 239 (TW). J Bacteriol 192:888-892. doi:10.1128/JB.01255-09

Huebner MDJ, DA Goldmann MD (1999) Coagulase-negative staphylococci: role as pathogens. Annu Rev Med 50:223-236. doi:10.1146/annurev.med.50.1.223 
Ibrahem S, Salmenlinna S, Virolainen A et al (2009) Carriage of methicillin-resistant staphylococci and their SCCmec types in a long-term-care facility. J Clin Microbiol 47:32-37. doi:10.1128/ JCM.01085-08

Kloos WE, Bannerman TL, Carolina N (1994) Update on clinical significance of coagulase-negative staphylococci. Clin Microbiol Rev 7:117-140

Kozitskaya S, Olson ME, Fey PD et al (2005) Clonal analysis of Staphylococcus epidermidis isolates carrying or lacking biofilmmediating genes by multilocus sequence typing. J Clin Microbiol 43:4751-4757. doi:10.1128/JCM.43.9.4751-4757.2005

Lebeaux D, Barbier F, Angebault C, Ben Mahdi L (2011) Evolution of methicillin-resistant coagulase-negative staphylococci nasal carriage in a remote population. Antimicrob Agents Chemother 56:1-35. doi:10.1128/AAC.00547-11

Li M, Du X, Villaruz AE et al (2012) MRSA epidemic linked to a quickly spreading colonization and virulence determinant. Nat Med 18:816-819. doi:10.1038/nm.2692.MRSA

Lo D, Pezina C, Muruet W et al (2010) Diversity of staphylococcal cassette chromosome mec structures in coagulase-negative staphylococci and relationship to drug resistance. J Med Microbiol 59:323-329. doi:10.1099/jmm.0.015800-0

Longauerova A (2006) Coagulase negative staphylococci and their participation in pathogenesis of human infections. Bratislava Med J 107:448-452

Maki H, Murakami K (1997) Formation of potent hybrid promoters of the mutant $1 \mathrm{~lm}$ Gene by IS 256 transposition in methicillinresistant Staphylococcus aureus. J Bacteriol 179:6944-6948

Mertens A, Ghebremedhin B (2013) Genetic determinants and biofilm formation of clinical Staphylococcus epidermidis isolates from blood cultures and indwelling devises. Eur J Microbiol Immunol 3:111-119. doi:10.1556/EuJMI.3.2013.2.4

Mombach Pinheiro Machado AB, Reiter KC, Paiva RM, Barth AL (2007) Distribution of staphylococcal cassette chromosome mec
(SCCmec) types I, II, III and IV in coagulase-negative staphylococci from patients attending a tertiary hospital in southern Brazil. J Med Microbiol 56:1328-1333. doi:10.1099/jmm.0. 47294-0

Otto M (2012) Insights \& perspectives coagulase-negative staphylococci as reservoirs of genes facilitating MRSA infection. BioEssays 35:4-11. doi:10.1002/bies.201200112

Rohde H, Kalitzky M, Kröger N et al (2004) Detection of virulenceassociated genes not useful for discriminating between invasive and commensal Staphylococcus epidermidis strains from a bone marrow transplant unit detection of virulence-associated genes not useful for discriminating between invasi. J Clin Microbiol 42:5614-5619. doi:10.1128/JCM.42.12.5614

Soumya KR, Mathew S, Sugathan S et al (2016) Studies on prevalence of biofilm associated genes and primary observation on sas X gene in clinical isolates of coagulase negative staphylococci (CoNS). APMIS 124:319-326. doi:10.1111/apm.12510

von Eiff C, Peters G, Heilmann C (2002) Review pathogenesis of infections due to coagulase- negative staphylococci. Lancet Infect Dis 2:677-685

Ziebuhr W, Heilmann C, Götz F et al (1997) Detection of the intercellular adhesion gene cluster (ica) and phase variation in Staphylococcus epidermidis blood culture strains and mucosal isolates. Detection of the intercellular adhesion gene cluster (ica) and phase variation in Staphylococcus. Infect Immun 63:890-896

Zong Z, Lü X (2010) Characterization of a new SCCmec element in Staphylococcus cohnii. PLoS One 5:1-6. doi:10.1371/journal. pone.0014016

Zong Z, Peng C, Lu X (2011) Diversity of SCCmec elements in methicillin-resistant coagulase-negative staphylococci clinical isolates. PLoS One 6:1-6. doi:10.1371/journal.pone.0020191 